\title{
HYBRID SYNCHRONIZATION OF LIU AND LÜ CHAOTIC Systems Via ADAPTIVE CONTROL
}

\author{
Sundarapandian Vaidyanathan ${ }^{1}$ \\ ${ }^{1}$ Research and Development Centre, Vel Tech Dr. RR \& Dr. SR Technical University \\ Avadi, Chennai-600 062, Tamil Nadu, INDIA \\ sundarvtu@gmail.com
}

\begin{abstract}
This paper derives new results for the hybrid synchronization of identical Liu systems, identical Lü systems, and non-identical Liu and Lü systems via adaptive control method. Liu system (Liu et al. 2004) and Lü system (Lü and Chen, 2002) are important models of three-dimensional chaotic systems. Hybrid synchronization of the three-dimensional chaotic systems addressed in this paper is achieved through the synchronization of the first and last pairs of states and anti-synchronization of the middle pairs of the two systems. Adaptive control method is deployed in this paper for the general case when the system parameters are unknown. Sufficient conditions for hybrid synchronization of identical Liu systems, identical Lü systems and non-identical Liu and Lü systems are derived via adaptive control theory and Lyapunov stability theory. Since the Lyapunov exponents are not needed for these calculations, the adaptive control method is very effective and convenient for the hybrid synchronization of the chaotic systems addressed in this paper. Numerical simulations are shown to illustrate the effectiveness of the proposed synchronization schemes.
\end{abstract}

\section{KEYWORDS}

Adaptive Control, Chaos, Hybrid Synchronization, Liu System, Lü System, Synchronization.

\section{INTRODUCTION}

A chaotic system is a very special nonlinear dynamical system, which has several properties such as the sensitivity to initial conditions as well as an irregular, unpredictable behaviour. This sensitivity to initial conditions of chaotic systems is popularly called as the butterfly effect [1].

Chaos is an interesting nonlinear phenomenon and has been extensively studied in the last two decades [1-40]. Chaos theory has been applied in many scientific disciplines such as Mathematics, Computer Science, Microbiology, Biology, Ecology, Economics, Population Dynamics and Robotics. Especially, chaos synchronization has found important applications in areas such as secure communications, data encryption, etc.

In 1990, Pecora and Carroll [2] deployed control techniques to synchronize two identical chaotic systems and showed that it was possible for some chaotic systems to be completely synchronized. From then on, chaos synchronization has been widely explored in a variety of fields including 
International Journal of Advanced Information Technology (IJAIT) Vol. 1, No. 6, December 2011

physical systems [3-4], chemical systems [5-6], ecological systems [7], secure communications [8-10], etc.

In most of the chaos synchronization approaches, the master-slave or drive-response formalism is used. If a particular chaotic system is called the master or drive system and another chaotic system is called the slave or response system, then the idea of the synchronization is to use the output of the master system to control the slave system so that the output of the slave system tracks the output of the master system asymptotically.

Since the seminal work by Pecora and Carroll [3], a variety of impressive approaches have been proposed for the synchronization of chaotic systems such as the OGY method [11], active control method [12-16], adaptive control method [17-22], sampled-data feedback synchronization method [23], time-delay feedback method [24], backstepping method [25-26], sliding mode control method [27-32], etc.

So far, many types of synchronization phenomenon have been presented such as complete synchronization [3], phase synchronization [33], generalized synchronization [34], antisynchronization [35-37], projective synchronization [38], generalized projective synchronization [39-40], etc.

Complete synchronization (CS) is characterized by the equality of state variables evolving in time, while anti-synchronization (AS) is characterized by the disappearance of the sum of relevant variables evolving in time.

Projective synchronization (PS) is characterized by the fact that the master and slave systems could be synchronized up to a scaling factor, whereas in generalized projective synchronization (GPS), the responses of the synchronized dynamical states synchronize up to a constant scaling matrix $\alpha$.

It is easy to see that the complete synchronization (CS) and anti-synchronization (AS) are special cases of the generalized projective synchronization (GPS) where the scaling matrix $\alpha=I$ and $\alpha=-I$, respectively.

In hybrid synchronization of chaotic systems [41-42], one part of the system is synchronized and the other part is anti-synchronized so that the complete synchronization (CS) and antisynchronization (AS) coexist in the system. The coexistence of CS and AS is highly useful in secure communication and chaotic encryption schemes.

In this paper, we investigate the hybrid chaos synchronization of uncertain three-dimensional chaotic systems, viz. identical Liu systems ([43], 2004), identical Lü systems ([44], 2002) and non-identical Liu and Lü systems. We consider the general case when the parameters of the systems are unknown.

This paper is organized as follows. In Section 2, we provide a description of the chaotic systems addressed in this paper, viz. Liu system (2005) and Lü system (2002). In Section 3, we discuss the hybrid synchronization of identical Liu systems via adaptive control. In Section 4, we discuss the hybrid synchronization of identical Lü systems via adaptive control. In Section 5, we discuss the hybrid chaos synchronization of identical Lü systems via adaptive control. Section 6 summarizes the main results obtained in this paper. 
International Journal of Advanced Information Technology (IJAIT) Vol. 1, No. 6, December 2011

\section{SYSTEMS DESCRIPTION}

The Liu system ([43], 2004) is described by the Liu dynamics

$$
\begin{aligned}
& \dot{x}_{1}=a\left(x_{2}-x_{1}\right) \\
& \dot{x}_{2}=b x_{1}-x_{1} x_{3} \\
& \dot{x}_{3}=-c x_{3}+d x_{1}^{2}
\end{aligned}
$$

where $x_{1}, x_{2}, x_{3}$ are the state variables and $a, b, c, d$ are positive, constant parameters of the system.

The Liu system (1) is chaotic when the parameter values are taken as

$$
a=10, \quad b=40, c=2.5 \text { and } d=4
$$

The state orbits of the Liu chaotic system (1) are shown in Figure 1.

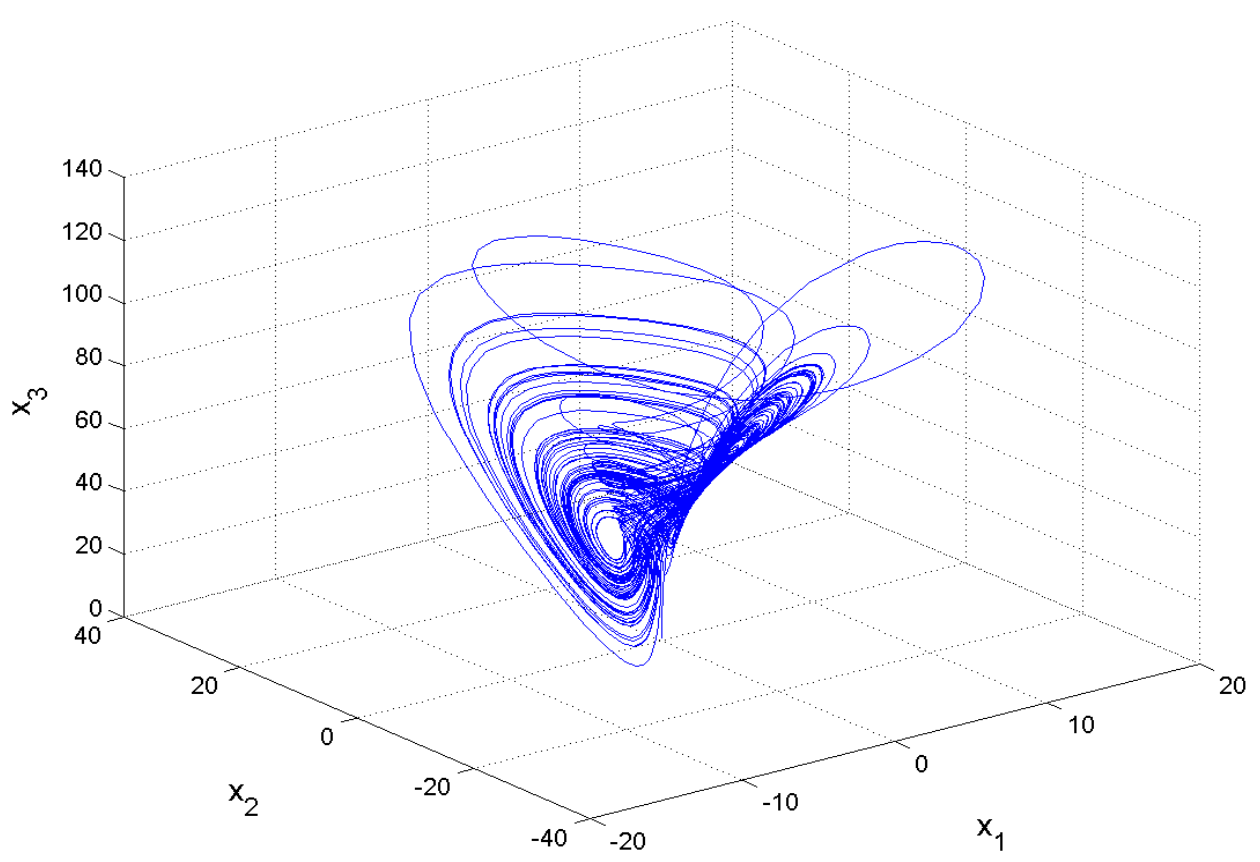

Figure 1. State Orbits of the Liu Chaotic System

The Lü system ([44], 2002) is described by

$$
\begin{aligned}
& \dot{x}_{1}=\alpha\left(x_{2}-x_{1}\right) \\
& \dot{x}_{2}=\gamma x_{2}-x_{1} x_{3} \\
& \dot{x}_{3}=-\beta x_{3}+x_{1} x_{2}
\end{aligned}
$$


International Journal of Advanced Information Technology (IJAIT) Vol. 1, No. 6, December 2011

where $x_{1}, x_{2}, x_{3}$ are the state variables and $\alpha, \beta, \gamma$ are positive constant parameters of the system.

The Lü system (2) is chaotic when the parameter values are taken as

$$
\alpha=36, \beta=3 \text { and } \gamma=20
$$

The state orbits of the Lü chaotic system (2) are shown in Figure 2.

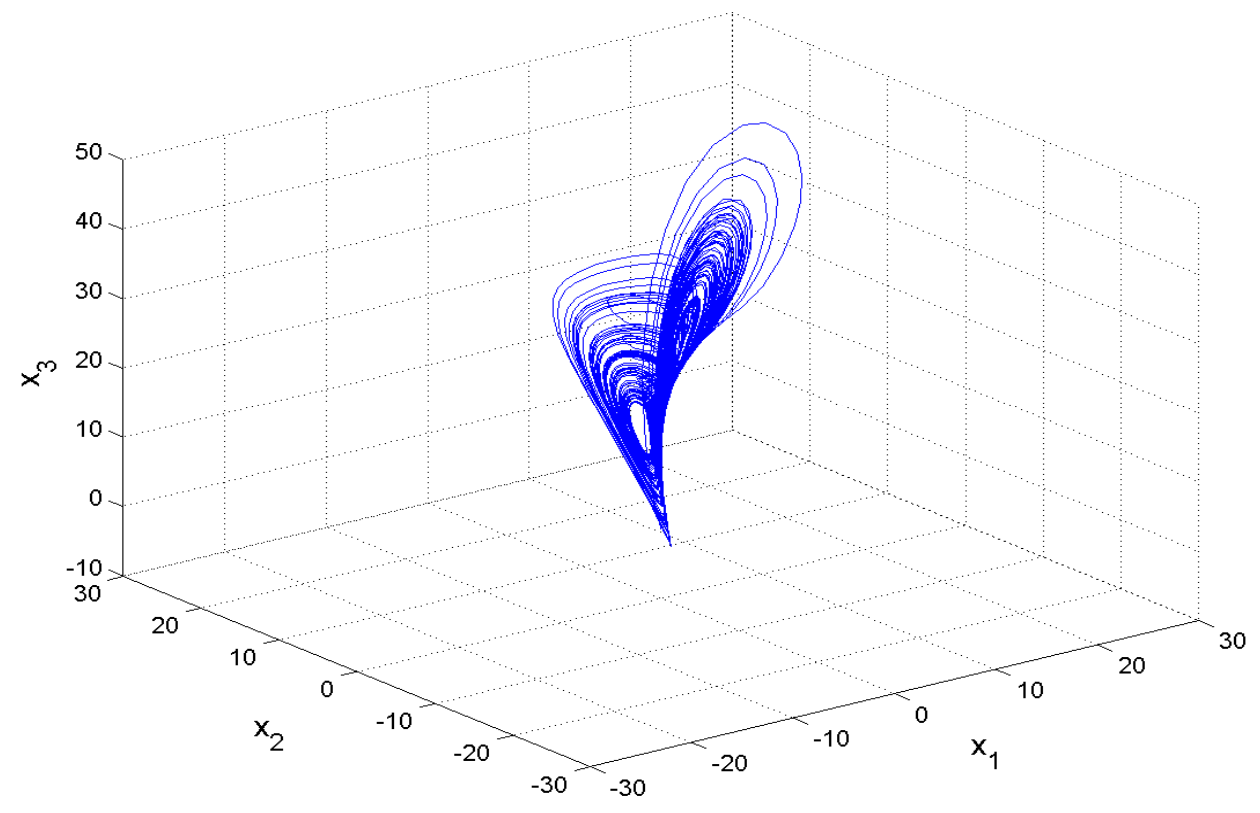

Figure 2. State Orbits of the Lü Chaotic System

\section{Hybrid Synchronization of Identical LiU Chaotic Systems Via ADAPTIVE CONTROL}

\subsection{Theoretical Results}

In this section, we discuss the hybrid synchronization of identical Liu chaotic systems ([43], 2004), where the parameters of the master and slave systems are unknown.

As the master system, we consider the Liu dynamics described by

$$
\begin{aligned}
& \dot{x}_{1}=a\left(x_{2}-x_{1}\right) \\
& \dot{x}_{2}=b x_{1}-x_{1} x_{3} \\
& \dot{x}_{3}=-c x_{3}+d x_{1}^{2}
\end{aligned}
$$

where $x_{1}, x_{2}, x_{3}$ are the state variables and $a, b, c, d$ are unknown, real, constant parameters of the system.

As the slave system, we consider the controlled Liu dynamics described by 


$$
\begin{aligned}
& \dot{y}_{1}=a\left(y_{2}-y_{1}\right)+u_{1} \\
& \dot{y}_{2}=b y_{1}-y_{1} y_{3}+u_{2} \\
& \dot{y}_{3}=-c y_{3}+d y_{1}^{2}+u_{3}
\end{aligned}
$$

where $y_{1}, y_{2}, y_{3}$ are the state variables and $u_{1}, u_{2}, u_{3}$ are the nonlinear controllers to be designed.

The hybrid chaos synchronization error is defined by

$$
\begin{aligned}
& e_{1}=y_{1}-x_{1} \\
& e_{2}=y_{2}+x_{2} \\
& e_{3}=y_{3}-x_{3}
\end{aligned}
$$

From the error equations (5), it is clear that one part of the two chaotic systems is completely synchronized (first and third states), while the other part is completely antisynchronized (second states) so that complete synchronization (CS) and antisynchronization (AS) coexist in the synchronization of the chaotic systems (3) and (4).

The error dynamics is easily obtained as

$$
\begin{aligned}
& \dot{e}_{1}=a\left(e_{2}-e_{1}-2 x_{2}\right)+u_{1} \\
& \dot{e}_{2}=b\left(e_{1}+2 x_{1}\right)-y_{1} y_{3}-x_{1} x_{3}+u_{2} \\
& \dot{e}_{3}=-c e_{3}+d\left(y_{1}^{2}-x_{1}^{2}\right)+u_{3}
\end{aligned}
$$

Let us now define the adaptive control functions

$$
\begin{aligned}
& u_{1}(t)=-\hat{a}\left(e_{2}-e_{1}-2 x_{2}\right)-k_{1} e_{1} \\
& u_{2}(t)=-\hat{b}\left(e_{1}+2 x_{1}\right)+y_{1} y_{3}+x_{1} x_{3}-k_{2} e_{2} \\
& u_{3}(t)=\hat{c} e_{3}-\hat{d}\left(y_{1}^{2}-x_{1}^{2}\right)-k_{3} e_{3}
\end{aligned}
$$

where $\hat{a}, \hat{b}, \hat{c}$ and $\hat{d}$ are estimates of $a, b, c$ and $d$, respectively, and $k_{i},(i=1,2,3)$ are positive constants.

Substituting (7) into (6), the error dynamics simplifies to

$$
\begin{aligned}
& \dot{e}_{1}=(a-\hat{a})\left(e_{2}-e_{1}-2 x_{2}\right)-k_{1} e_{1} \\
& \dot{e}_{2}=(b-\hat{b})\left(e_{1}+2 x_{1}\right)-k_{2} e_{2} \\
& \dot{e}_{3}=-(c-\hat{c}) e_{3}+(d-\hat{d})\left(y_{1}^{2}-x_{1}^{2}\right)-k_{3} e_{3}
\end{aligned}
$$

Let us now define the parameter estimation errors as

$$
e_{a}=a-\hat{a}, e_{b}=b-\hat{b}, e_{c}=c-\hat{c} \text { and } e_{d}=d-\hat{d}
$$

Substituting (9) into (8), we obtain the error dynamics as 
International Journal of Advanced Information Technology (IJAIT) Vol. 1, No. 6, December 2011

$$
\begin{aligned}
& \dot{e}_{1}=e_{a}\left(e_{2}-e_{1}-2 x_{2}\right)-k_{1} e_{1} \\
& \dot{e}_{2}=e_{b}\left(e_{1}+2 x_{1}\right)-k_{2} e_{2} \\
& \dot{e}_{3}=-e_{c} e_{3}+e_{d}\left(y_{1}^{2}-x_{1}^{2}\right)-k_{3} e_{3}
\end{aligned}
$$

For the derivation of the update law for adjusting the estimates of the parameters, the Lyapunov approach is used.

We consider the quadratic Lyapunov function defined by

$$
V\left(e_{1}, e_{2}, e_{3}, e_{a}, e_{b}, e_{c}, e_{d}\right)=\frac{1}{2}\left(e_{1}^{2}+e_{2}^{2}+e_{3}^{2}+e_{a}^{2}+e_{b}^{2}+e_{c}^{2}+e_{d}^{2}\right),
$$

which is a positive definite function on $R^{7}$.

We also note that

$$
\dot{e}_{a}=-\dot{\hat{a}}, \dot{e}_{b}=-\dot{\hat{b}}, \quad \dot{e}_{c}=-\dot{\hat{c}} \quad \text { and } \quad \dot{e}_{d}=-\dot{\hat{d}}
$$

Differentiating (11) along the trajectories of (10) and using (12), we obtain

$$
\begin{aligned}
\dot{V}= & -k_{1} e_{1}^{2}-k_{2} e_{2}^{2}-k_{3} e_{3}^{2}+e_{a}\left[e_{1}\left(e_{2}-e_{1}-2 x_{2}\right)-\dot{\hat{a}}\right]+e_{b}\left[e_{2}\left(e_{1}+2 x_{1}\right)-\dot{\hat{b}}\right] \\
& +e_{c}\left[-e_{3}^{2}-\dot{\hat{c}}\right]+e_{d}\left[e_{3}\left(y_{1}^{2}-x_{1}^{2}\right)-\dot{\hat{d}}\right]
\end{aligned}
$$

In view of Eq. (13), the estimated parameters are updated by the following law:

$$
\begin{aligned}
& \dot{\hat{a}}=e_{1}\left(e_{2}-e_{1}-2 x_{2}\right)+k_{4} e_{a} \\
& \dot{\hat{b}}=e_{2}\left(e_{1}+2 x_{1}\right)+k_{5} e_{b} \\
& \dot{\hat{c}}=-e_{3}^{2}+k_{6} e_{c} \\
& \dot{\hat{d}}=e_{3}\left(y_{1}^{2}-x_{1}^{2}\right)+k_{7} e_{d}
\end{aligned}
$$

where $k_{4}, k_{5}, k_{6}$ and $k_{7}$ are positive constants.

Substituting (14) into (13), we obtain

$$
\dot{V}=-k_{1} e_{1}^{2}-k_{2} e_{2}^{2}-k_{3} e_{3}^{2}-k_{4} e_{a}^{2}-k_{5} e_{b}^{2}-k_{6} e_{c}^{2}-k_{7} e_{d}^{2}
$$

which is a negative definite function on $R^{7}$.

Thus, by Lyapunov stability theory [45], it is immediate that the hybrid synchronization error $e_{i},(i=1,2,3)$ and the parameter estimation error $e_{a}, e_{b}, e_{c}, e_{d}$ decay to zero exponentially with time.

Hence, we have proved the following result. 
International Journal of Advanced Information Technology (IJAIT) Vol. 1, No. 6, December 2011

Theorem 1. The identical Liu chaotic systems (3) and (4) with unknown parameters are globally and exponentially hybrid synchronized via the adaptive control law (7), where the update law for the parameter estimates is given by (14) and $k_{i},(i=1,2, \ldots, 7)$ are positive constants. Also, the parameter estimates $\hat{a}(t), \hat{b}(t), \hat{c}(t)$ and $\hat{d}(t)$ exponentially converge to the original values of the parameters $a, b, c$ and $d$, respectively, as $t \rightarrow \infty$.

\subsection{Numerical Results}

For the numerical simulations, the fourth-order Runge-Kutta method with time-step $h=10^{-6}$ is used to solve the 3-D chaotic systems (3) and (4) with the adaptive control law (14) and the parameter update law (14) using MATLAB.

We take

$$
k_{i}=4 \text { for } i=1,2, \ldots, 7 \text {. }
$$

For the Liu chaotic systems (3) and (4), the parameter values are taken as

$$
a=10, \quad b=40, \quad c=2.5, \quad d=4
$$

Suppose that the initial values of the parameter estimates are

$$
\hat{a}(0)=4, \hat{b}(0)=12, \hat{c}(0)=8, \hat{d}(0)=10 .
$$

The initial values of the master system (3) are taken as

$$
x_{1}(0)=7, \quad x_{2}(0)=11, \quad x_{3}(0)=15
$$

The initial values of the slave system (4) are taken as

$$
y_{1}(0)=30, \quad y_{2}(0)=18, \quad y_{3}(0)=26
$$

Figure 3 depicts the hybrid-synchronization of the identical Liu chaotic systems (3) and (4). It may also be noted that the odd states of the two systems are completely synchronized, while the even states of the two systems are anti-synchronized.

Figure 4 shows that the estimated values of the parameters, viz. $\hat{a}(t), \hat{b}(t), \hat{c}(t)$ and $\hat{d}(t)$ converge exponentially to the system parameters

$$
a=10, b=40, c=2.5 \text { and } d=4
$$

as $t$ tends to infinity. 
International Journal of Advanced Information Technology (IJAIT) Vol. 1, No. 6, December 2011
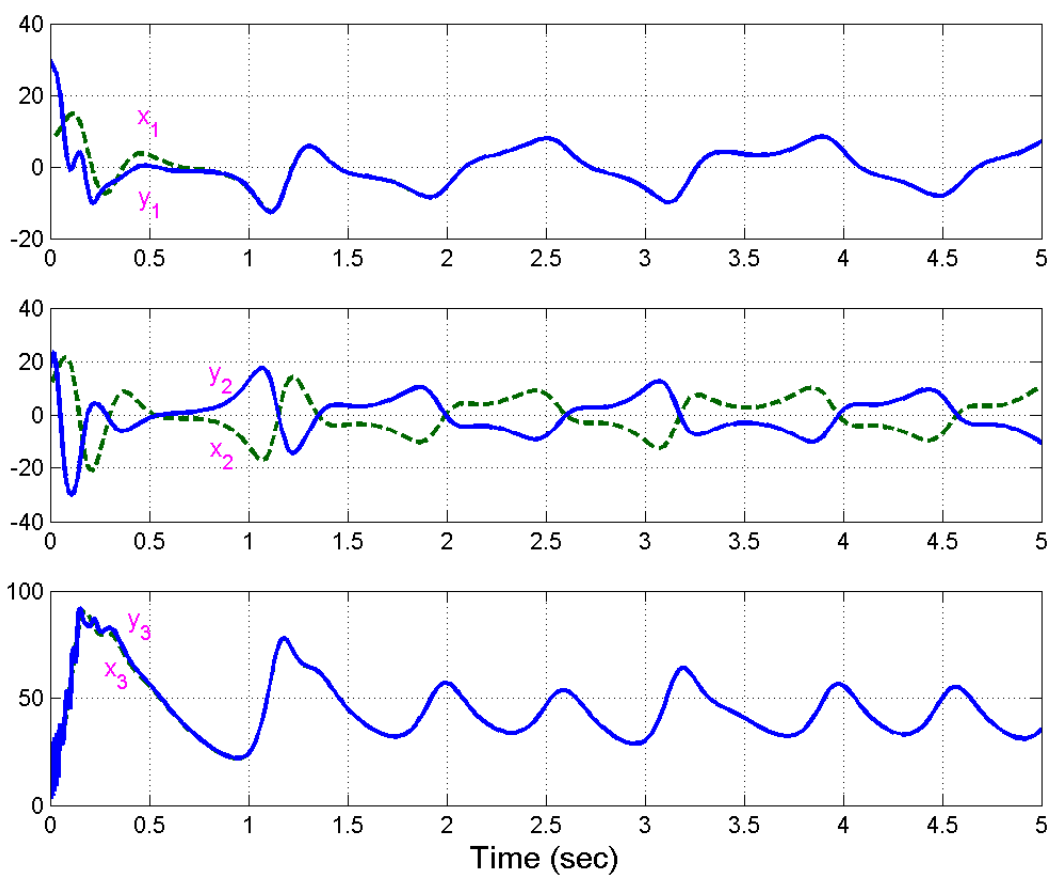

Figure 3. Hybrid-Synchronization of Liu Chaotic Systems

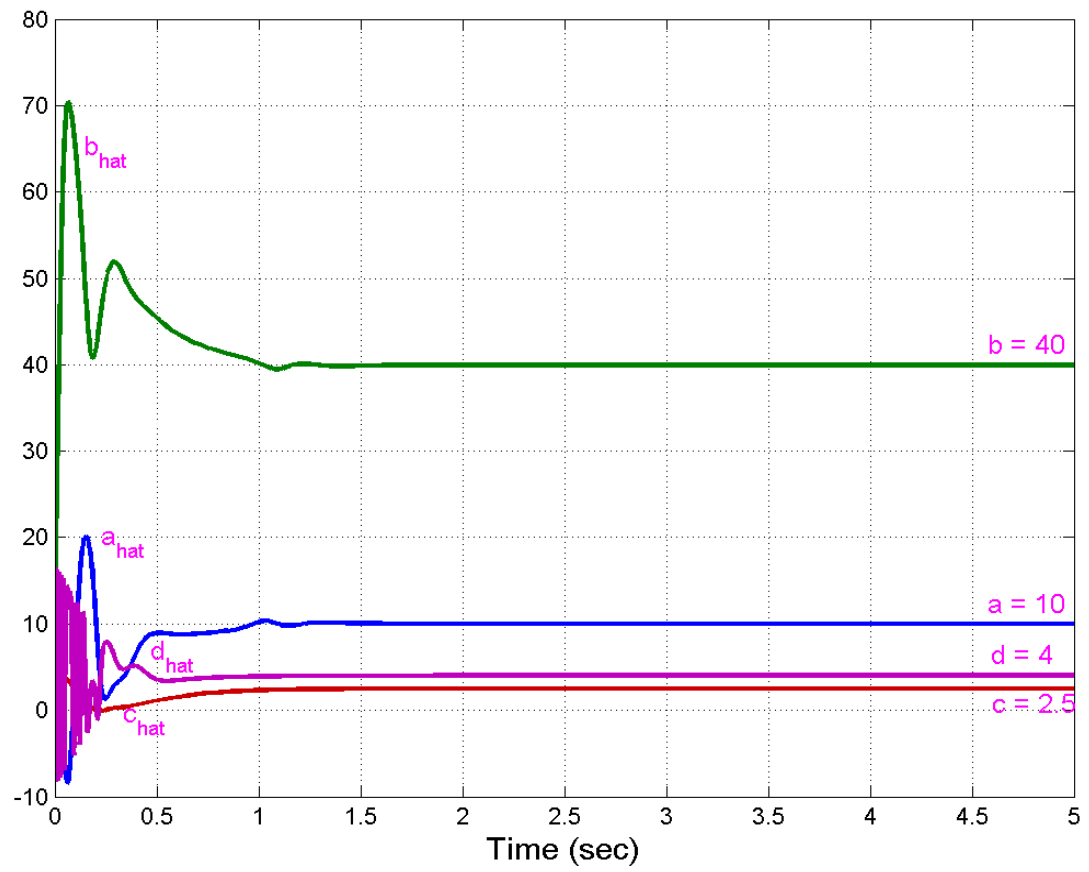

Figure 4. Parameter Estimates $\hat{a}(t), \hat{b}(t), \hat{c}(t), \hat{d}(t)$ 
International Journal of Advanced Information Technology (IJAIT) Vol. 1, No. 6, December 2011

\section{Hybrid Synchronization of Identical Lü ChaOtic Systems via ADAPTIVE CONTROL}

\subsection{Theoretical Results}

In this section, we discuss the hybrid synchronization of identical Lü chaotic systems ([44], 2002), where the parameters of the master and slave systems are unknown.

As the master system, we consider the Lü dynamics described by

$$
\begin{aligned}
& \dot{x}_{1}=\alpha\left(x_{2}-x_{1}\right) \\
& \dot{x}_{2}=\gamma x_{2}-x_{1} x_{3} \\
& \dot{x}_{3}=-\beta x_{3}+x_{1} x_{2}
\end{aligned}
$$

where $x_{1}, x_{2}, x_{3}$ are the state variables and $\alpha, \beta, \gamma$ are unknown, real ,constant parameters of the system.

As the slave system, we consider the controlled Lü dynamics described by

$$
\begin{aligned}
& \dot{y}_{1}=\alpha\left(y_{2}-y_{1}\right)+u_{1} \\
& \dot{y}_{2}=\gamma y_{2}-y_{1} y_{3}+u_{2} \\
& \dot{y}_{3}=-\beta y_{3}+y_{1} y_{2}+u_{3}
\end{aligned}
$$

where $y_{1}, y_{2}, y_{3}$ are the state variables and $u_{1}, u_{2}, u_{3}$ are the nonlinear controllers to be designed.

The hybrid chaos synchronization error is defined by

$$
\begin{aligned}
& e_{1}=y_{1}-x_{1} \\
& e_{2}=y_{2}+x_{2} \\
& e_{3}=y_{3}-x_{3}
\end{aligned}
$$

From the error equations (18), it is clear that one part of the two chaotic systems is completely synchronized (first and third states), while the other part is completely antisynchronized (second states) so that complete synchronization (CS) and antisynchronization (AS) coexist in the synchronization of the chaotic systems (16) and (17).

The error dynamics is easily obtained as

$$
\begin{aligned}
& \dot{e}_{1}=\alpha\left(e_{2}-e_{1}-2 x_{2}\right)+u_{1} \\
& \dot{e}_{2}=\gamma e_{2}-y_{1} y_{3}-x_{1} x_{3}+u_{2} \\
& \dot{e}_{3}=-\beta e_{3}+y_{1} y_{2}-x_{1} x_{2}+u_{3}
\end{aligned}
$$

Let us now define the adaptive control functions 
International Journal of Advanced Information Technology (IJAIT) Vol. 1, No. 6, December 2011

$$
\begin{aligned}
& u_{1}(t)=-\hat{\alpha}\left(e_{2}-e_{1}-2 x_{2}\right)-k_{1} e_{1} \\
& u_{2}(t)=-\hat{\gamma} e_{2}+y_{1} y_{3}+x_{1} x_{3}-k_{2} e_{2} \\
& u_{3}(t)=\hat{\beta} e_{3}-y_{1} y_{2}+x_{1} x_{2}-k_{3} e_{3}
\end{aligned}
$$

where $\hat{\alpha}, \hat{\beta}$ and $\hat{\gamma}$ are estimates of $\alpha, \beta$ and $\gamma$, respectively, and $k_{i},(i=1,2,3)$ are positive constants.

Substituting (20) into (19), the error dynamics simplifies to

$$
\begin{aligned}
& \dot{e}_{1}=(\alpha-\hat{\alpha})\left(e_{2}-e_{1}-2 x_{2}\right)-k_{1} e_{1} \\
& \dot{e}_{2}=(\gamma-\hat{\gamma}) e_{2}-k_{2} e_{2} \\
& \dot{e}_{3}=-(\beta-\hat{\beta}) e_{3}-k_{3} e_{3}
\end{aligned}
$$

Let us now define the parameter estimation errors as

$$
\begin{aligned}
& e_{\alpha}=\alpha-\hat{\alpha} \\
& e_{\beta}=\beta-\hat{\beta} \\
& e_{\gamma}=\gamma-\hat{\gamma}
\end{aligned}
$$

Substituting (22) into (21), we obtain the error dynamics as

$$
\begin{aligned}
& \dot{e}_{1}=e_{\alpha}\left(e_{2}-e_{1}-2 x_{2}\right)-k_{1} e_{1} \\
& \dot{e}_{2}=e_{\gamma} e_{2}-k_{2} e_{2} \\
& \dot{e}_{3}=-e_{\beta} e_{3}-k_{3} e_{3}
\end{aligned}
$$

For the derivation of the update law for adjusting the estimates of the parameters, the Lyapunov approach is used.

We consider the quadratic Lyapunov function defined by

$$
V\left(e_{1}, e_{2}, e_{3}, e_{\alpha}, e_{\beta}, e_{\gamma}\right)=\frac{1}{2}\left(e_{1}^{2}+e_{2}^{2}+e_{3}^{2}+e_{\alpha}^{2}+e_{\beta}^{2}+e_{\gamma}^{2}\right)
$$

which is a positive definite function on $R^{6}$.

We also note that

$$
\begin{aligned}
& \dot{e}_{\alpha}=-\dot{\hat{\alpha}} \\
& \dot{e}_{\beta}=-\dot{\hat{\beta}} \\
& \dot{e}_{\gamma}=-\dot{\hat{\gamma}}
\end{aligned}
$$

Differentiating (24) along the trajectories of (23) and using (25), we obtain 
International Journal of Advanced Information Technology (IJAIT) Vol. 1, No. 6, December 2011

$$
\begin{aligned}
\dot{V}= & -k_{1} e_{1}^{2}-k_{2} e_{2}^{2}-k_{3} e_{3}^{2}+e_{\alpha}\left[e_{1}\left(e_{2}-e_{1}-2 x_{2}\right)-\dot{\hat{\alpha}}\right]+e_{\beta}\left[-e_{3}^{2}-\dot{\hat{\beta}}\right] \\
& +e_{\gamma}\left[e_{2}^{2}-\dot{\hat{\gamma}}\right]
\end{aligned}
$$

In view of Eq. (26), the estimated parameters are updated by the following law:

$$
\begin{aligned}
& \dot{\hat{\alpha}}=e_{1}\left(e_{2}-e_{1}-2 x_{2}\right)+k_{4} e_{\alpha} \\
& \dot{\hat{\beta}}=-e_{3}^{2}+k_{5} e_{\beta} \\
& \dot{\hat{\gamma}}=e_{2}^{2}+k_{6} e_{\gamma}
\end{aligned}
$$

where $k_{4}, k_{5}$ and $k_{6}$ are positive constants.

Substituting (27) into (26), we obtain

$$
\dot{V}=-k_{1} e_{1}^{2}-k_{2} e_{2}^{2}-k_{3} e_{3}^{2}-k_{4} e_{\alpha}^{2}-k_{5} e_{\beta}^{2}-k_{6} e_{\gamma}^{2}
$$

which is a negative definite function on $R^{6}$.

Thus, by Lyapunov stability theory [45], it is immediate that the hybrid synchronization error $e_{i},(i=1,2,3)$ and the parameter estimation error $e_{\alpha}, e_{\beta}, e_{\gamma}$ decay to zero exponentially with time.

Hence, we have proved the following result.

Theorem 2. The identical Lü chaotic systems (16) and (17) with unknown parameters are globally and exponentially hybrid synchronized via the adaptive control law (20), where the update law for the parameter estimates is given by (27) and $k_{i},(i=1,2, \ldots, 6)$ are positive

constants. Also, the parameter estimates $\hat{\alpha}(t), \hat{\beta}(t)$ and $\hat{\gamma}(t)$ exponentially converge to the original values of the parameters $\alpha, \beta$ and $\gamma$, respectively, as $t \rightarrow \infty$.

\subsection{Numerical Results}

For the numerical simulations, the fourth-order Runge-Kutta method with time-step $h=10^{-6}$ is used to solve the 3-D chaotic systems (16) and (17) with the adaptive control law (20) and the parameter update law (27) using MATLAB.

We take $k_{i}=4$ for $i=1,2, \ldots, 6$.

For the Lü chaotic systems (16) and (17), the parameter values are taken as

$$
\alpha=36, \quad \beta=3, \quad \gamma=20
$$

Suppose that the initial values of the parameter estimates are

$$
\hat{\alpha}(0)=9, \quad \hat{\beta}(0)=24, \hat{\gamma}(0)=11
$$

The initial values of the master system (16) are taken as 
International Journal of Advanced Information Technology (IJAIT) Vol. 1, No. 6, December 2011

$$
x_{1}(0)=22, \quad x_{2}(0)=14, \quad x_{3}(0)=30
$$

The initial values of the slave system (17) are taken as

$$
y_{1}(0)=10, \quad y_{2}(0)=28, \quad y_{3}(0)=7
$$

Figure 5 depicts the hybrid-synchronization of the identical Lü chaotic systems (16) and (17). It may also be noted that the odd states of the two systems are completely synchronized, while the even states of the two systems are anti-synchronized.

Figure 6 shows that the estimated values of the parameters, viz. $\hat{\alpha}(t), \hat{\beta}(t)$ and $\hat{\gamma}(t)$ converge exponentially to the system parameters $\alpha=36, \beta=3$ and $\gamma=20$, respectively, as $t \rightarrow \infty$.
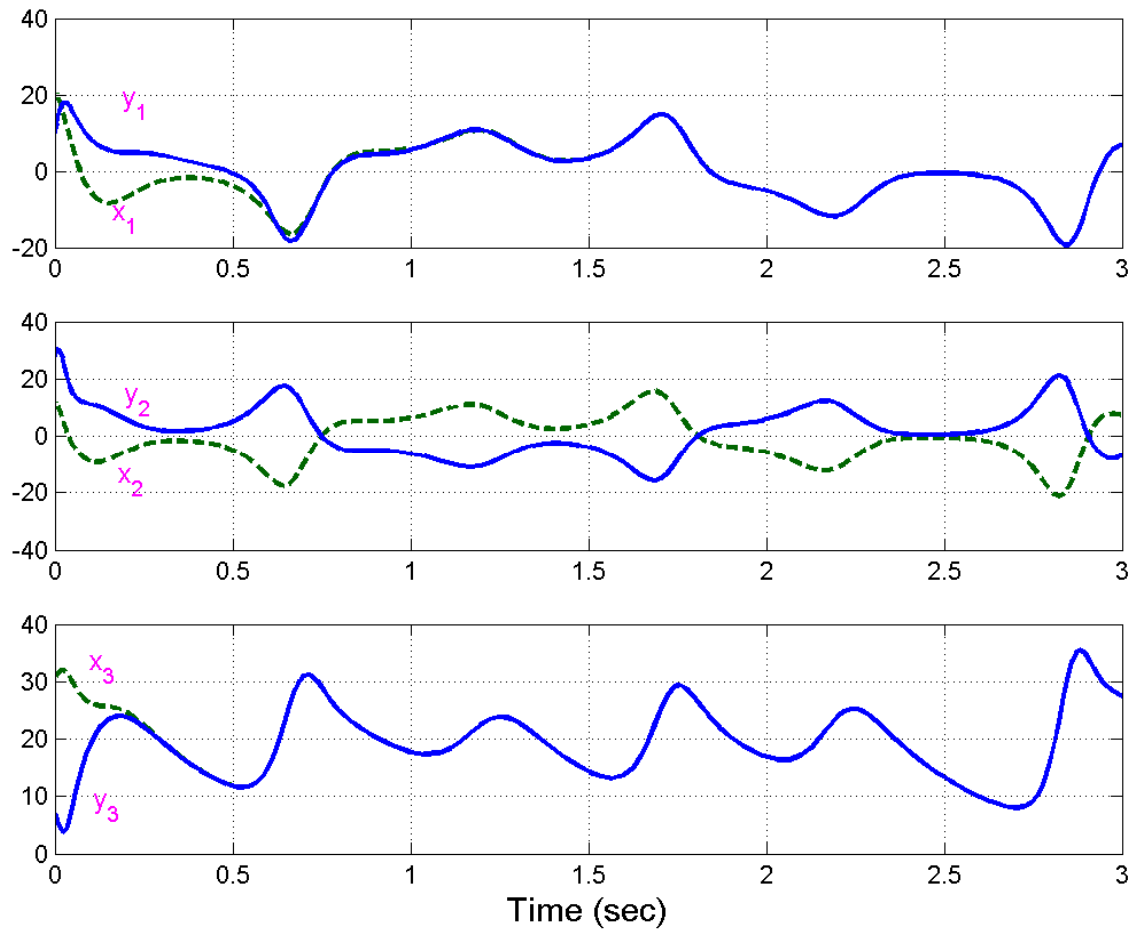

Figure 5. Hybrid-Synchronization of Lü Chaotic Systems 


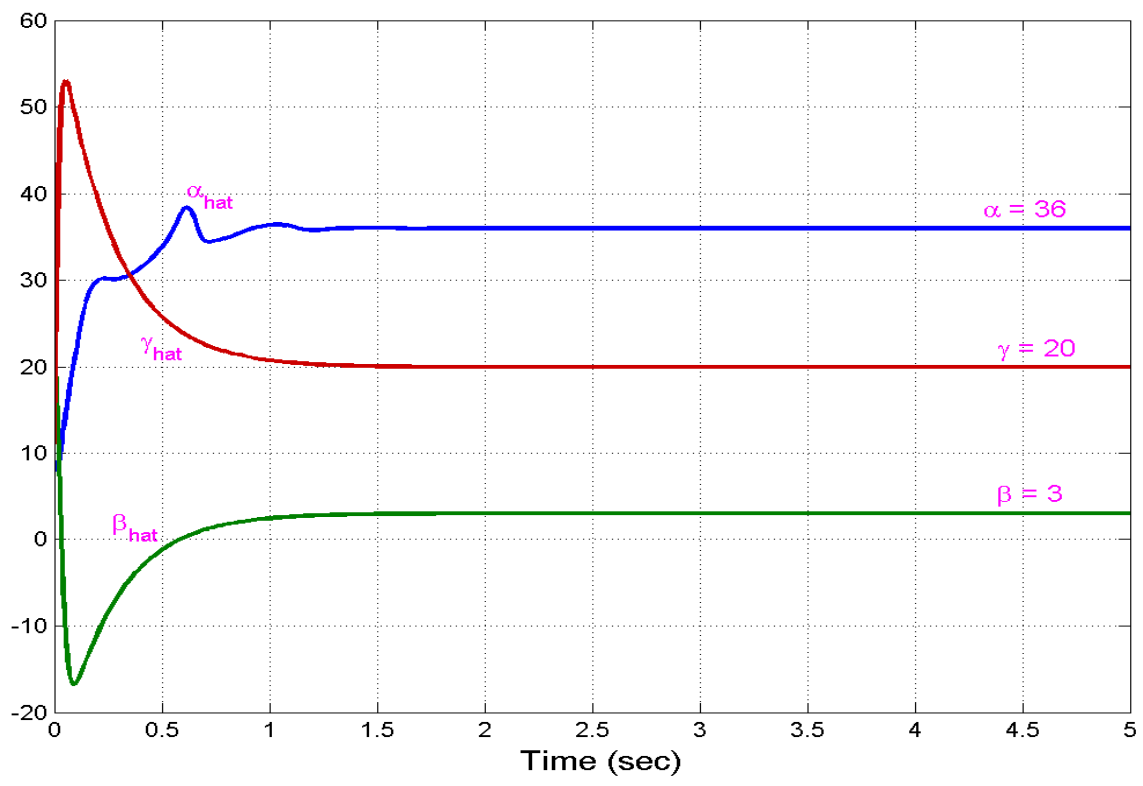

10

\section{Hybrid Synchronization of LiU ANd Lü Chaotic Systems via ADAPTIVE CONTROL}

\subsection{Theoretical Results}

In this section, we discuss the hybrid synchronization of identical Lü chaotic systems ([44], 2002), where the parameters of the master and slave systems are unknown.

As the master system, we consider the Liu dynamics described by

$$
\begin{aligned}
& \dot{x}_{1}=a\left(x_{2}-x_{1}\right) \\
& \dot{x}_{2}=b x_{1}-x_{1} x_{3} \\
& \dot{x}_{3}=-c x_{3}+d x_{1}^{2}
\end{aligned}
$$

where $x_{1}, x_{2}, x_{3}$ are the state variables and $a, b, c, d$ are unknown, real ,constant parameters of the system.

As the slave system, we consider the controlled Lü dynamics described by

$$
\begin{aligned}
& \dot{y}_{1}=\alpha\left(y_{2}-y_{1}\right)+u_{1} \\
& \dot{y}_{2}=\gamma y_{2}-y_{1} y_{3}+u_{2} \\
& \dot{y}_{3}=-\beta y_{3}+y_{1} y_{2}+u_{3}
\end{aligned}
$$

where $y_{1}, y_{2}, y_{3}$ are the state variables, $\alpha, \beta, \gamma$ are unknown, real, constant parameters of the system and $u_{1}, u_{2}, u_{3}$ are the nonlinear controllers to be designed. 
The hybrid chaos synchronization error is defined by

$$
\begin{aligned}
& e_{1}=y_{1}-x_{1} \\
& e_{2}=y_{2}+x_{2} \\
& e_{3}=y_{3}-x_{3}
\end{aligned}
$$

From the error equations (31), it is clear that one part of the two chaotic systems is completely synchronized (first and third states), while the other part is completely antisynchronized (second states) so that complete synchronization (CS) and antisynchronization (AS) coexist in the synchronization of the chaotic systems (29) and (30).

The error dynamics is easily obtained as

$$
\begin{aligned}
& \dot{e}_{1}=\alpha\left(y_{2}-y_{1}\right)-a\left(x_{2}-x_{1}\right)+u_{1} \\
& \dot{e}_{2}=\gamma y_{2}+b x_{1}-y_{1} y_{3}-x_{1} x_{3}+u_{2} \\
& \dot{e}_{3}=-\beta y_{3}+c x_{3}+y_{1} y_{2}-d x_{1}^{2}+u_{3}
\end{aligned}
$$

Let us now define the adaptive control functions

$$
\begin{aligned}
& u_{1}(t)=-\hat{\alpha}\left(y_{2}-y_{1}\right)+\hat{a}\left(x_{2}-x_{1}\right)-k_{1} e_{1} \\
& u_{2}(t)=-\hat{\gamma} y_{2}-\hat{b} x_{1}+y_{1} y_{3}+x_{1} x_{3}-k_{2} e_{2} \\
& u_{3}(t)=\hat{\beta} y_{3}-\hat{c} x_{3}+\hat{d} x_{1}^{2}-y_{1} y_{2}-k_{3} e_{3}
\end{aligned}
$$

where $\hat{a}, \hat{b}, \hat{c}, \hat{d}, \hat{\alpha}, \hat{\beta}$ and $\quad \hat{\gamma}$ are estimates of $a, b, c, d, \alpha, \beta$ and $\gamma$, respectively, and $k_{i},(i=1,2,3)$ are positive constants.

Substituting (33) into (32), the error dynamics simplifies to

$$
\begin{aligned}
& \dot{e}_{1}=(\alpha-\hat{\alpha})\left(y_{2}-y_{1}\right)-(a-\hat{a})\left(x_{2}-x_{1}\right)-k_{1} e_{1} \\
& \dot{e}_{2}=(\gamma-\hat{\gamma}) y_{2}+(b-\hat{b}) x_{1}-k_{2} e_{2} \\
& \dot{e}_{3}=-(\beta-\hat{\beta}) y_{3}+(c-\hat{c}) x_{3}-(d-\hat{d}) x_{1}^{2}-k_{3} e_{3}
\end{aligned}
$$

Let us now define the parameter estimation errors as

$$
\begin{aligned}
& e_{a}=a-\hat{a}, \quad e_{b}=b-\hat{b}, \quad e_{c}=c-\hat{c}, \quad e_{d}=d-\hat{d} \\
& e_{\alpha}=\alpha-\hat{\alpha}, \quad e_{\beta}=\beta-\hat{\beta}, \quad e_{\gamma}=\gamma-\hat{\gamma}
\end{aligned}
$$

Substituting (35) into (34), we obtain the error dynamics as

$$
\begin{aligned}
& \dot{e}_{1}=e_{\alpha}\left(y_{2}-y_{1}\right)-e_{a}\left(x_{2}-x_{1}\right)-k_{1} e_{1} \\
& \dot{e}_{2}=e_{\gamma} y_{2}+e_{b} x_{1}-k_{2} e_{2} \\
& \dot{e}_{3}=-e_{\beta} y_{3}+e_{c} x_{3}-e_{d} x_{1}^{2}-k_{3} e_{3}
\end{aligned}
$$


International Journal of Advanced Information Technology (IJAIT) Vol. 1, No. 6, December 2011

For the derivation of the update law for adjusting the estimates of the parameters, the Lyapunov approach is used.

We consider the quadratic Lyapunov function defined by

$$
V=\frac{1}{2}\left(e_{1}^{2}+e_{2}^{2}+e_{3}^{2}+e_{a}^{2}+e_{b}^{2}+e_{c}^{2}+e_{d}^{2}+e_{\alpha}^{2}+e_{\beta}^{2}+e_{\gamma}^{2}\right)
$$

which is a positive definite function on $R^{10}$.

We also note that

$$
\begin{aligned}
& \dot{e}_{a}=-\dot{\hat{a}}, \quad \dot{e}_{b}=-\dot{\hat{b}}, \quad \dot{e}_{c}=-\dot{\hat{c}}, \quad \dot{e}_{d}=-\dot{\hat{d}} \\
& \dot{e}_{\alpha}=-\dot{\hat{\alpha}}, \dot{e}_{\beta}=-\dot{\hat{\beta}}, \dot{e}_{\gamma}=-\dot{\hat{\gamma}}
\end{aligned}
$$

Differentiating (37) along the trajectories of (36) and using (38), we obtain

$$
\begin{aligned}
\dot{V}=- & k_{1} e_{1}^{2}-k_{2} e_{2}^{2}-k_{3} e_{3}^{2}+e_{a}\left[-e_{1}\left(x_{2}-x_{1}\right)-\dot{\hat{a}}\right]+e_{b}\left[e_{2} x_{1}-\dot{\hat{b}}\right]+e_{c}\left[e_{3} x_{3}-\dot{\hat{c}}\right] \\
& +e_{d}\left[-e_{3} x_{1}^{2}-\dot{\hat{d}}\right]+e_{\alpha}\left[e_{1}\left(y_{2}-y_{1}\right)-\dot{\hat{\alpha}}\right]+e_{\beta}\left[-e_{3} y_{3}-\dot{\hat{\beta}}\right]+e_{\gamma}\left[e_{2} y_{2}-\dot{\hat{\gamma}}\right]
\end{aligned}
$$

In view of Eq. (39), the estimated parameters are updated by the following law:

$$
\begin{array}{ll}
\dot{\hat{a}}=-e_{1}\left(x_{2}-x_{1}\right)+k_{4} e_{a}, & \dot{\hat{\alpha}}=e_{1}\left(y_{2}-y_{1}\right)+k_{8} e_{\alpha} \\
\dot{\hat{b}}=e_{2} x_{1}+k_{5} e_{b}, & \dot{\hat{\beta}}=-e_{3} y_{3}+k_{9} e_{\beta} \\
\dot{\hat{c}}=e_{3} x_{3}+k_{6} e_{c}, & \dot{\hat{\gamma}}=e_{2} y_{2}+k_{10} e_{\gamma} \\
\dot{\hat{d}}=-e_{3} x_{1}^{2}+k_{7} e_{d} &
\end{array}
$$

where $k_{i},(i=4, \ldots, 10)$ are positive constants.

Substituting (40) into (39), we obtain

$$
\dot{V}=-k_{1} e_{1}^{2}-k_{2} e_{2}^{2}-k_{3} e_{3}^{2}-k_{4} e_{a}^{2}-k_{5} e_{b}^{2}-k_{6} e_{c}^{2}-k_{7} e_{d}^{2}-k_{8} e_{\alpha}^{2}-k_{9} e_{\beta}^{2}-k_{10} e_{\gamma}^{2}
$$

which is a negative definite function on $R^{10}$.

Thus, by Lyapunov stability theory [45], it is immediate that the hybrid synchronization error $e_{i},(i=1,2,3)$ and the parameter estimation error $e_{a}, e_{b}, e_{c}, e_{d}, e_{\alpha}, e_{\beta}, e_{\gamma}$ decay to zero exponentially with time.

Hence, we have proved the following result.

Theorem 3. The non-identical Liu system (29) and Lü system (30) with unknown parameters are globally and exponentially hybrid synchronized via the adaptive control law (33), where the 
International Journal of Advanced Information Technology (IJAIT) Vol. 1, No. 6, December 2011

update law for the parameter estimates is given by (40) and $k_{i},(i=1,2, \ldots, 10)$ are positive constants. Also, the parameter estimates $\hat{a}(t), \hat{b}(t), \hat{c}(t), \hat{d}(t), \hat{\alpha}(t), \hat{\beta}(t)$ and $\hat{\gamma}(t)$ exponentially converge to the original values of the parameters $a, b, c, d, \alpha, \beta$ and $\gamma$, respectively, as $t \rightarrow \infty$.

\subsection{Numerical Results}

For the numerical simulations, the fourth-order Runge-Kutta method with time-step $h=10^{-6}$ is used to solve the 3-D chaotic systems (29) and (30) with the adaptive control law (33) and the parameter update law (40) using MATLAB.

We take $k_{i}=4$ for $i=1,2, \ldots, 10$.

For the Liu chaotic system, the parameter values are taken as

$$
a=10, b=40, c=2.5, \quad d=4
$$

For the Lü chaotic system, the parameter values are taken as

$$
\alpha=36, \quad \beta=3, \quad \gamma=20
$$

Suppose that the initial values of the parameter estimates are

$$
\hat{a}(0)=7, \hat{b}(0)=15, \hat{c}(0)=20, \hat{d}(0)=9, \hat{\alpha}(0)=12, \hat{\beta}(0)=6, \hat{\gamma}(0)=5
$$

The initial values of the master system (29) are taken as

$$
x_{1}(0)=17, \quad x_{2}(0)=11, \quad x_{3}(0)=28
$$

The initial values of the slave system (30) are taken as

$$
y_{1}(0)=30, \quad y_{2}(0)=16, \quad y_{3}(0)=12
$$

Figure 7 depicts the hybrid-synchronization of the non-identical Liu system (29) and Lü chaotic system (30). It may also be noted that the odd states of the two systems are completely synchronized, while the even states of the two systems are anti-synchronized.

Figure 8 shows that the estimated values of the parameters, viz. $\hat{a}(t), \hat{b}(t), \hat{c}(t), \hat{d}(t), \hat{\alpha}(t)$, $\hat{\beta}(t)$ and $\hat{\gamma}(t)$ converge exponentially to the system parameters $a=10, b=40, c=2.5$, $d=4, \alpha=36, \beta=3$ and $\gamma=20$, respectively, as $t \rightarrow \infty$. 

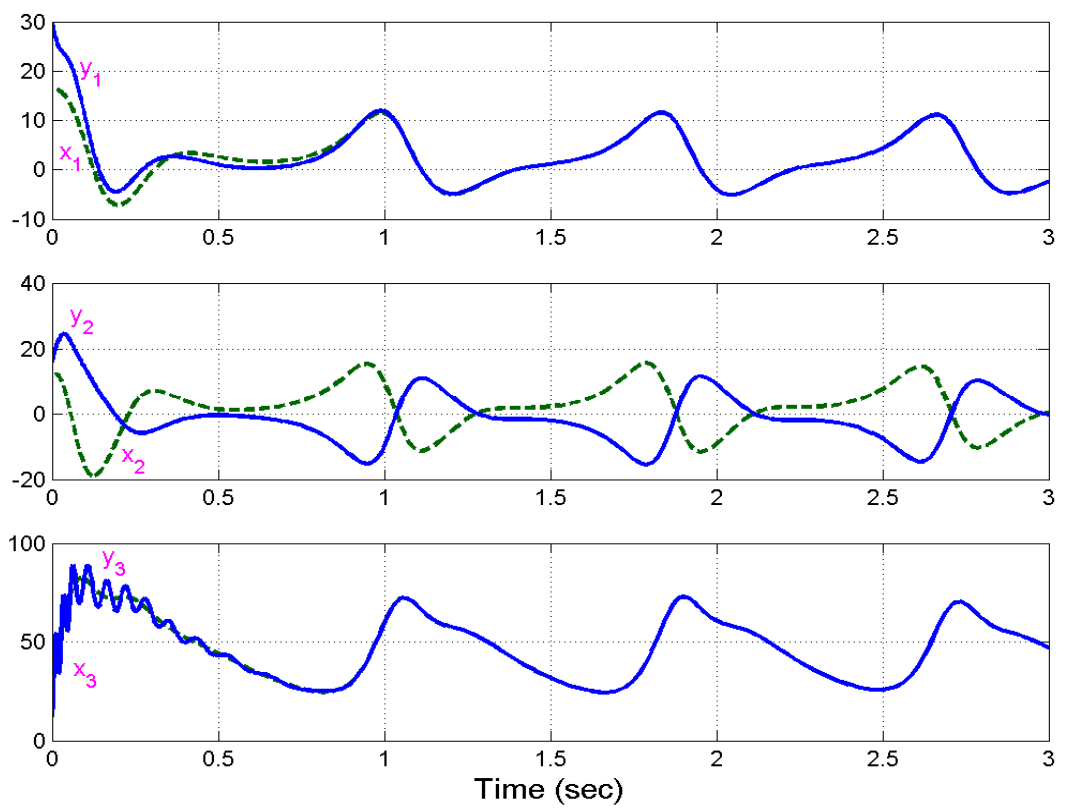

Figure 7. Hybrid-Synchronization of Liu and Lü Chaotic Systems

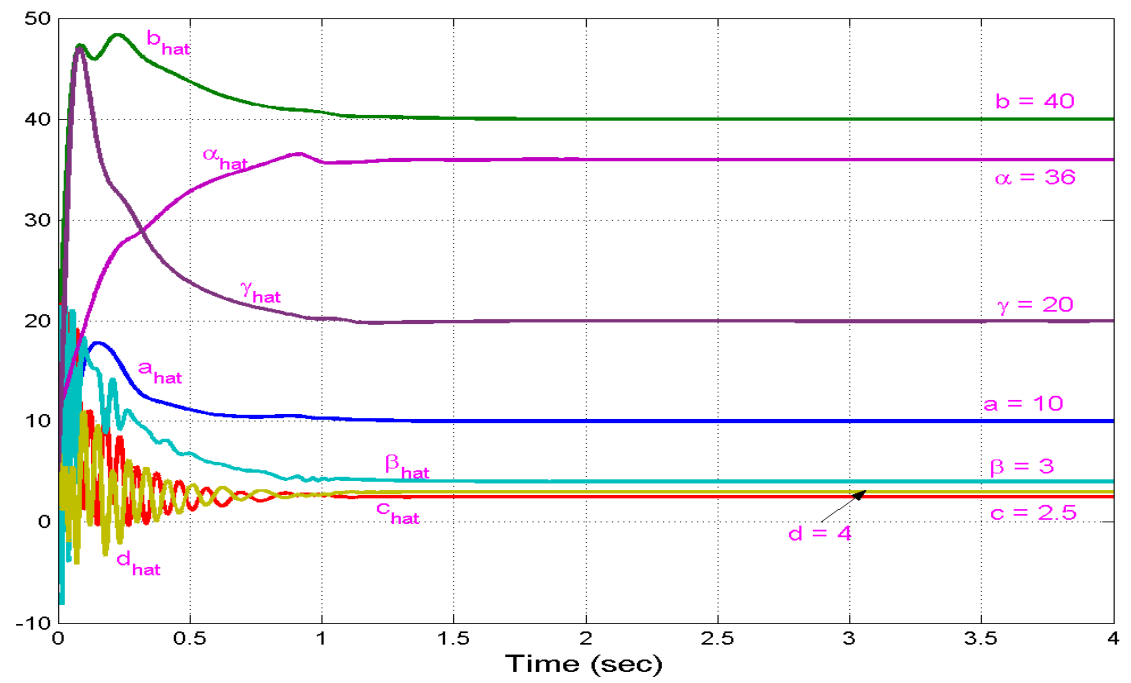

Figure 8. Parameter Estimates $\hat{a}(t), \hat{b}(t), \hat{c}(t), \hat{d}(t), \hat{\alpha}(t), \hat{\beta}(t), \hat{\gamma}(t)$

\section{Conclusions}

In this paper, we have deployed adaptive control method to derive new results for the hybrid synchronization of identical Liu systems (2004), identical Lü systems (2002) and non-identical Liu and Lü systems with unknown parameters. The hybrid synchronization results derived in this paper are established using Lyapunov stability theory. Since the Lyapunov exponents are not 
International Journal of Advanced Information Technology (IJAIT) Vol. 1, No. 6, December 2011

required for these calculations, the adaptive control method is a very effective and convenient for achieving hybrid chaos synchronization for the uncertain three-dimensional chaotic systems discussed in this paper. Numerical simulations are presented to demonstrate the effectiveness of the adaptive synchronization schemes derived in this paper for the hybrid chaos synchronization of identical and non-identical uncertain Liu and Lü systems.

\section{REFERENCES}

[1] Alligood, K.T., Sauer, T. \& Yorke, J.A. (1997) Chaos: An Introduction to Dynamical Systems, Springer, New York.

[2] Pecora, L.M. \& Carroll, T.L. (1990) "Synchronization in chaotic systems," Physical Review Letters, Vol. 64, pp 821-824.

[3] Fabiny, L. \& Wiesenfield, K. (1991) "Clustering behaviour of oscillator arrays," Physical Review A, Vol. 43, pp 2640-2648.

[4] Lakshmanan, M. \& Murali, K. (1996) Nonlinear Oscillators: Controlling and Synchronization, World Scientific, Singapore.

[5] Han, S.K., Kerrer, C. \& Kuramoto, Y. (1995) "Dephasing and bursting in coupled neural oscillators," Physical Review Letters, Vol. 75, pp 3190-3193.

[6] Niu, H., Zhang, Q. \& Zhang, Y. (2002) "The chaos synchronization of a singular chemical model and a Williamowski-Rossler model," International Journal of Information and Systems Sciences, Vol. 6, No. 4, pp 355-364.

[7] Blasius, B., Huppert, A. \& Stone, L. (1999) "Complex dynamics and phase synchronization in spatially extended ecological system”, Nature, Vol. 399, pp 354-359.

[8] Kocarev, L. \& Parlitz, U. (1995) "General approach for chaotic synchronization with applications to communication,” Physical Review Letters, Vol. 74, pp 5028-5030.

[9] Boccaletti, S., Farini, A. \& Arecchi, F.T. (1997) "Adaptive synchronization of chaos for secure communication," Physical Review E, Vol. 55, No. 5,

[10] Tao, Y. (1999) "Chaotic secure communication systems - history and new results," Telecommun. Review, Vol. 9, pp 597-634.

[11] Ott, E., Grebogi, C. \& Yorke, J.A. (1990) “Controlling chaos”, Physical Review Letters, Vol. 64, pp 1196-1199.

[12] Ho, M.C. \& Hung, Y.C. (2002) "Synchronization of two different chaotic systems using generalized active network," Physics Letters A, Vol. 301, pp 424-428.

[13] Huang, L., Feng, R. \& Wang, M. (2005) "Synchronization of chaotic systems via nonlinear control," Physical Letters A, Vol. 320, pp 271-275.

[14] Chen, H.K. (2005) "Global chaos synchronization of new chaotic systems via nonlinear control," Chaos, Solitons \& Fractals, Vol. 23, pp 1245-1251.

[15] Sundarapandian, V. \& Suresh, R. (2011) "Global chaos synchronization of hyperchaotic Qi and Jia systems by nonlinear control," International Journal of Distributed and Parallel Systems, Vol. 2, No. 2, pp 83-94.

[16] Sundarapandian, V. \& Karthikeyan, R. (2011) "Active controller design for global chaos antisynchronization of Li and Tigan chaotic systems," International Journal of Information Technology and Computer Science, vol. 3, no. 4, pp 255-268.

[17] Lu, J., Wu, X., Han, X. \& Lü, J. (2004) "Adaptive feedback synchronization of a unified chaotic system," Physics Letters A, Vol. 329, pp 327-333.

[18] Sundarapandian, V. (2011) "Adaptive control and synchronization of hyperchaotic Liu system," International Journal of Computer Science, Engineering and Information Technology, Vol. 1, No. 2, pp 29-40.

[19] Sundarapandian, V. (2011) "Adaptive control and synchronization of hyperchaotic Newton-Leipnik system," International Journal of Advanced Information Technology, Vol. 1, No. 3, pp 22-33.

[20] Sundarapandian, V. (2011) "Adaptive synchronization of hyperchaotic Lorenz and hyperchaotic Lü systems," International Journal of Instrumentation and Control Systems, Vol. 1, No. 1, pp 1-18.

[21] Sundarapandian, V. (2011) "Adaptive control and synchronization of hyperchaotic Cai system," International Journal of Control Theory and Computer Modeling, Vol. 1, No. 1, pp 1-13. 
International Journal of Advanced Information Technology (IJAIT) Vol. 1, No. 6, December 2011

[22] Sundarapandian, V. (2011) "Adaptive control and synchronization of Liu's four-wing chaotic system with cubic nonlinearity," International Journal of Computer Science, Engineering and Applications, Vol. 1, No. 4, pp 127-138.

[23] Zhao, J. \& Lu, J. (2006) "Using sampled-data feedback control and linear feedback synchronization in a new hyperchaotic system," Chaos, Solitons and Fractals, Vol. 35, pp 376-382.

[24] Park, J.H. \& Kwon, O.M. (2003) "A novel criterion for delayed feedback control of time-delay chaotic systems," Chaos, Solitons \& Fractals, Vol. 17, pp 709-716.

[25] Tan, X., Zhang, J. \& Yang, Y. (2003) "Synchronizing chaotic systems using backstepping design," Chaos, Solitons \& Fractals, Vol. 16, pp. 37-45.

[26] Vincent, U.E. (2008) "Chaotic synchronization using active control and backstepping control: A comparative analysis," Nonlinear Analysis: Modelling and Control, Vol. 13, No. 2, pp 253-261.

[27] Slotine, J.E. \& Sastry, S.S. (1983) "Tracking control of nonlinear systems using sliding surface with application to robotic manipulators," Internat. J. Control, Vol. 38, pp 465-492.

[28] Utkin, V.I. (1993) "Sliding mode control design principles and applications to electric drives," IEEE Trans. Industrial Electronics, Vol. 40, pp 23-36, 1993.

[29] Sundarapandian, V. \& S. Sivaperumal (2011) "Anti-synchronization of hyperchaotic Lorenz systems by sliding mode control," International Journal on Computer Science and Engineering, Vol. 3, No. 6, pp 2438-2449.

[30] Sundarapandian, V. (2011) "Sliding mode controller design for synchronization of Shimizu-Morioka chaotic systems," International Journal of Information Sciences and Techniques, Vol. 1, No. 1, pp 20-29.

[31] Sundarapandian, V. (2011) "Global chaos synchronization of four-wing chaotic systems by sliding mode control," International Journal of Control Theory and Computer Modeling, Vol. 1, No. 1, pp 15-31.

[32] Sundarapandian, V. (2011) "Global chaos synchronization of hyperchaotic Newton-Leipnik systems by sliding mode control," International Journal of Information Technology, Convergence and Services, Vol. 1, No. 4, pp 34-43.

[33] Ge, Z.M. \& Chen, C.C. (2004) "Phase synchronization of coupled chaotic multiple time scales systems", Chaos, Solitons and Fractals, Vol. 20, pp 639-647.

[34] Wang, Y.W. \& Guan, Z.H. (2006) "Generalized synchronization of continuous chaotic systems", Chaos, Solitons and Fractals, Vol. 27, pp 97-101.

[35] Zhang, X. \& Zhu, H. (2008) "Anti-synchronization of two different hyperchaotic systems via active and adaptive control," International Journal of Nonlinear Science, Vol. 6, pp 216-223.

[36] Chiang, T., Lin, J., Liao, T. \& Yan, J. (2008) "Anti-synchronization of uncertain unified chaotic systems with dead-zone nonlinearity," Nonlinear Analysis: Modelling and Control, Vol. 68, pp 26292637.

[37] Sundarapandian, V. \& Karthikeyan, R. (2011) “Anti-synchronization of Pan and Liu chaotic systems by active nonlinear control," International Journal of Engineering Science and Technology, Vol. 3, No. 5, pp 3596-3604.

[38] Qiang, J. (2007) "Projective synchronization of a new hyperchaotic Lorenz system", Phys. Lett. A, Vol. 370, pp 40-45.

[39] Jian-Ping, Y. \& Chang-Pin, L. (2006) "Generalized projective synchronization for the chaotic Lorenz system and the chaotic Chen system”, J. Shanghai Univ., Vol. 10, pp 299-304.

[40] Li, R.H., Xu, W. \& Li, S. (2007) "Adaptive generalized projective synchronization in different chaotic systems based on parameter identification", Phys. Lett. A, Vol. 367, pp 199-206.

[41] Sundarapandian, V. (2011) "Hybrid synchronization of Harb and Wang chaotic systems by active nonlinear control," International Journal of Computer Information Systems, Vol. 3, No. 2, pp 7-12.

[42] Sundarapandian, V. (2011) "Hybrid synchronization of Lorenz and Pehlivan chaotic systems by active nonlinear control," International Journal of Advances in Science and Technology, Vol. 3, No. 2, pp 7-12.

[43] Liu, C., Liu, T., Liu, L. \& Liu, K. (2004) “A new chaotic attractor," Chaos, Solitons and Fractals, Vol. 22, pp 1031-1038.

[44] Lü, J. \& Chen, G. (2002) “A new chaotic attractor joined,” International Journal of Bifurcation and Chaos, Vol. 12, pp 659-661.

[45] Hahn, W. (1967) The Stability of Motion, Springer, New York. 
International Journal of Advanced Information Technology (IJAIT) Vol. 1, No. 6, December 2011

\section{Author}

Dr. V. Sundarapandian obtained his Doctor of Science degree in Electrical and Systems Engineering from Washington University, Saint Louis, USA under the guidance of Late Dr. Christopher I. Byrnes (Dean, School of Engineering and Applied Science) in 1996. He is currently Professor in the Research and Development Centre at Vel Tech Dr. RR \& Dr. SR Technical University, Chennai, Tamil Nadu, India. He has published over 190 refereed international publications. He has published over 100 papers in National Conferences and over 50 papers in International Conferences. He is the Editor-in-Chief of International Journal of Mathematics and Scientific Computing, International Journal of Instrumentation and Control Systems, International Journal of Control Systems and Computer Modelling, and International Journal of Information Technology, Control and Automation. He is an Associate Editor of the International Journals - International Journal of Control Theory and Applications, International Journal of Information Sciences and Techniques, Journal of Electronics and Electrical Engineering, International Journal of Computer Information Systems, International Journal of Advances in Science and Technology, etc. His research interests are Linear and Nonlinear Control Systems, Chaos Theory and Control, Soft Computing, Optimal Control, Process Control, Operations Research, Mathematical Modelling, Scientific Computing etc. He has delivered several Key Note Lectures on Linear and Nonlinear Control Systems, Chaos Theory and Control, MATLAB, SCILAB, etc. 